\title{
Resolving the one-dimensional autonomous flow-free explosion problem
}

\author{
James T. Murphy III* \\ Faculty advisor: Gautam Iyer ${ }^{\dagger}$ \\ Department of Mathematical Sciences \\ Carnegie Mellon University
}

July 25, 2014

\begin{abstract}
We introduce the explosion problem for semilinear elliptic and parabolic PDEs. The explosion problem is a question of existence of nonnegative solutions to $-L \phi=\lambda g(x, \phi)$ and nonnegative global solutions to $\partial_{t} \phi-L \phi=\lambda g(x, t, \phi)$. Under certain conditions there is a $\lambda^{*}>0$, called the explosion threshold, for which $\lambda<\lambda^{*}$ implies a solution exists, and $\lambda>\lambda^{*}$ implies no solution exists. In this paper we focus on the one-dimensional elliptic case. Our main result is the explicit calculation of the explosion threshold $\lambda^{*}$ for the one-dimensional autonomous flow-free problem $-\phi^{\prime \prime}=\lambda g(\phi)$.
\end{abstract}

Acknowledgment: We thank the National Science Foundation (grant DMS-1252912) and the Alfred P. Sloan foundation for their support.

\section{Contents}

1 Introduction $\quad 220$

1.1 The explosion problem . . . . . . . . . . . . . . . . . . . . . . 220

2 The one-dimensional flow-free case $-\phi^{\prime \prime}=\lambda g(\phi) \quad 222$

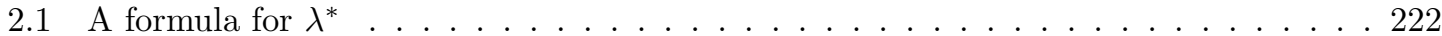

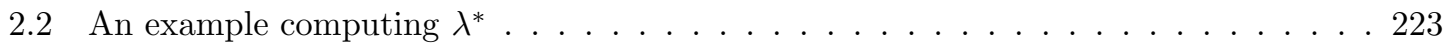

2.3 An example with no solution for $\lambda=\lambda^{*} \ldots \ldots \ldots \ldots$. . . . . . . . . . 224

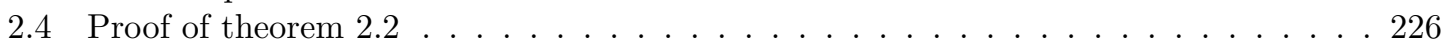

3 References $\quad 231$

*James T. Murphy III, Department of Mathematical Sciences, Carnegie Mellon University, james@intfxdx.com

†Gautam Iyer, Department of Mathematical Sciences, Carnegie Mellon University, gautam@math.cmu.edu 


\section{Introduction}

\subsection{The explosion problem}

In its most general form, the explosion problem is a question about positive solutions to semilinear elliptic PDEs of the form

$$
\left\{\begin{array}{cl}
-L \phi=\lambda g(x, \phi) & x \in \Omega \\
\phi(x)=f(x) & x \in \partial \Omega
\end{array}\right.
$$

and parabolic PDEs, possibly with different types of boundary conditions, of the form

$$
\left\{\begin{array}{cl}
\partial_{t} \phi-L \phi=\lambda g(x, t, \phi) & (x, t) \in \Omega \times(0, T) \\
\phi(x, 0)=f(x) & (x, 0) \in \Omega \times\{0\} \\
\phi(x, t)=b(x, t) & (x, t) \in \partial \Omega \times(0, T) .
\end{array}\right.
$$

We would like to understand under what conditions these PDEs have solutions, have unique, maximal, or minimal solutions, what kind of regularity solutions have, and fast numerical schemes to compute solutions when they exist.

An interesting observation is that PDEs of the form (1.1) or (1.2) often exhibit dichotomous behavior depending on whether the positive parameter $\lambda$ is large or small. In particular, under certain conditions, there exists a critical $\lambda^{*}>0$, called the explosion threshold, such that

(a) in the elliptic case, for all $\lambda<\lambda^{*}$ the problem (1.1) has at least one nonnegative solution, while for all $\lambda>\lambda^{*}$ there are no nonnegative solutions to (1.1), and

(b) in the parabolic case, for all $\lambda<\lambda^{*}$ the problem (1.2) has at least one nonnegative solution which is global in time $(T=\infty)$, while for all $\lambda>\lambda^{*}$, any solution to (1.2) blows up in finite time.

Later we will be precise about the exact assumptions we will need for the one-dimensional case. For now, we provide generic assumptions which are commonly seen for the higher-dimensional case. Frequently, $\Omega$ is taken to be a bounded, open, simply connected domain in $\mathbb{R}^{d}$ with smooth boundary. Positivity, monotonicity, and convexity assumptions are made on $g$, as well as fast growth conditions. For the case $g=g(\phi)$, fast growth usually means one of $g^{\prime}(s) \rightarrow \infty, g(s) / s \rightarrow \infty$, or $\int_{0}^{\infty} \frac{1}{g(s)} d s<\infty$, and the canonical examples to keep in mind are $g(s):=e^{s}$ and $g(s):=(1+s)^{p}$ for $p>1$. The linear operator $L$ is typically taken uniformly elliptic of the form $L:=\triangle-u \cdot \nabla$ for a smooth divergence-free vector field $u$ which is tangential to $\partial \Omega$. In this situation, one may view the elliptic and parabolic problems respectively as steady-state and time-dependent advection-diffusion systems in an incompressible fluid with nonlinear source terms.

The existence of the critical $\lambda^{*}$ for the elliptic case was largely developed by Keener and Keller [16], Joseph and Lundgren [13], and Crandall and Rabinowitz [5]. As for the parabolic case, Sattinger showed in [22] the relationship between lower and upper solutions of the elliptic problem and global solutions to the parabolic problem. Namely, if $\phi$ and $\bar{\phi}$ are lower and upper solutions to the elliptic problem respectively, then for any continuous initial data $\varphi$ with $\phi \leq \varphi \leq \bar{\phi}$, there is a global solution to the parabolic problem with initial data $\varphi$ which stays bounded between $\underline{\phi}$ and $\bar{\phi}$. Moreover, [22] also showed that with zero boundary conditions, if there is a global solution $\phi$ to the parabolic problem with initial data $\varphi:=\underline{\phi}$, where $\phi$ is a lower solution to the elliptic 
problem, then $\phi \nearrow \tilde{\phi}$ as $t \rightarrow \infty$, where $\tilde{\phi}$ is a solution to the elliptic problem. Note that since $g$ is assumed to be positive, $\phi:=0$ will always be a lower solution to the elliptic problem, so the previous statement can at least be applied in this case.

More recently, effort has been spent to understand how mixing with different incompressible flows affects the explosion threshold. Intuitively, the explosion threshold exists because hotspots develop in the fluid. When $\lambda$ is large, these hotspots become so hot that their existence would imply that the solution to the elliptic problem must be $\phi:=+\infty$ everywhere. This also gives reason to why we focus mainly on divergence-free flows. If we were allowed to compress our fluid towards a point, surely we could make a hotspot as hot as we want. Amongst incompressible flows however, compression towards a point is not possible since divergence-free flows preserve volume.

Furthermore, stirring by an incompressible flow $u$ is actually known to improve diffusivity in the sense that the principal eigenvalue $\mu_{1}[u]$ of the problem

$$
\left\{\begin{array}{cl}
-\triangle \phi^{u}+u \cdot \nabla \phi^{u}=\mu_{1}[u] \phi^{u} & x \in \Omega \\
\phi^{u}(x)=0 & x \in \partial \Omega
\end{array}\right.
$$

is never larger than the corresponding $\mu_{1}[0]$ for $u:=0$. From this intuition we may expect that the explosion threshold $\lambda^{*}(u)$ with stirring by an incompressible $u$ is always larger than the explosion threshold $\lambda^{*}(0)$ with no stirring. Novikov showed in [19] that when $\Omega$ is a ball, indeed $\lambda^{*}(0) \leq \lambda^{*}(u)$ for incompressible flows $u$. Surprisingly, this does not appear to be the case when $\Omega$ is not a ball. Kagan et. al. showed numerically in [14] that in a long thin rectangle with $g(s):=e^{s}$ there is an incompressible $u$ with $\lambda^{*}(u)<\lambda^{*}(0)$. Furthermore, Iyer et. al. showed in [12] that with $g(s):=1$, unless $\Omega$ is a ball, there is an incompressible $u$ which creates hotspots in the sense that $\left\|\phi^{u}\right\|_{\infty}>\left\|\phi^{0}\right\|_{\infty}$. In [3], Berestycki et. al. studied how much stirring can change the explosion threshold. In particular, they presented bounds on $\lambda^{*}(u)$ that are independent of the advecting flow $u$, and they characterized when the explosion threshold tends to $\infty$ under fast stirring.

In this paper, we take steps towards explicitly computing the explosion threshold by looking at the one-dimensional autonomous flow-free case $-\phi^{\prime \prime}=\lambda g(\phi)$. In this specific case, we will be able to completely characterize solutions to the differential equation, and doing so will allow us to give a formula for the explosion threshold $\lambda^{*}$. It is our hope that the ideas in this paper will be generalized or applied in such a way as to uncover a formula for $\lambda^{*}$ in the higher-dimensional cases. 


\section{The one-dimensional flow-free case $-\phi^{\prime \prime}=\lambda g(\phi)$}

\subsection{A formula for $\lambda^{*}$}

We direct our attention to the one-dimensional case. Say $\Omega:=(a, b)$ with $a<b$. In one dimension, the only divergence-free $u$ are constants, and of these only $u:=0$ is tangential to $\partial \Omega$. Hence we will consider the explosion problem for

$$
\left\{\begin{array}{cl}
-\phi^{\prime \prime}=\lambda g(\phi) & x \in(a, b) \\
\phi(x)=0 & x \in\{a, b\}
\end{array}\right.
$$

We claim that we can go so far as to provide the form of any nonnegative solution $\phi$ of (2.1), if one exists, for any non-constant, continuous, increasing, convex $g:[0, \infty) \rightarrow(0, \infty)$, and that this will allow us to explicitly compute $\lambda^{*}$. First we need a regularity lemma about solutions to (2.1), then we state the main theorem.

Lemma 2.1. Any solution $\phi$ of (2.1) satisfies $\phi \in C^{2}([a, b])$ so that $-\phi^{\prime \prime}=\lambda g(\phi)$ holds on $[a, b]$. Moreover, if $g \in C^{k}([0, \infty))$ for $k \geq 0$, then $\phi \in C^{k+2}([a, b])$. The only assumption on $g$ we require for this lemma is continuity.

Theorem 2.2. There is $\lambda^{*}>0$ such that for all $\lambda<\lambda^{*}$ the problem (2.1) has a minimal nonnegative solution, and for all $\lambda>\lambda^{*}$ the problem (2.1) has no nonnegative solutions. Furthermore, $\lambda^{*}$ is given by

$$
\lambda^{*}=\sup _{k>0} \frac{2}{(b-a)^{2}} H_{k}\left(G^{-1}(k)\right)^{2}
$$

where

$$
G(u):=\int_{0}^{u} g(s) d s, \quad H_{k}(y):=\int_{0}^{y} \frac{d u}{\sqrt{k-G(u)}},
$$

and when $\lambda \leq \lambda^{*}$, $\phi$ solves (2.1) if and only if $\phi$ is given by

$$
\phi(x):=H_{k}^{-1}(\sqrt{2 \lambda} T(x))
$$

where

$$
T(x):=\frac{b-a}{2}-\left|x-\frac{b+a}{2}\right|
$$

and $k>0$ is a solution to

$$
\lambda=\frac{2}{(b-a)^{2}} H_{k}\left(G^{-1}(k)\right)^{2} .
$$

Moreover, all nonnegative solutions $\phi$ are in $C^{2}([a, b])$, and if the right derivative $g_{+}^{\prime}(s) \rightarrow \infty$ as $s \rightarrow \infty$, then a nonnegative solution with $\lambda=\lambda^{*}$ exists.

Theorem 2.2 gives us both a method of computing $\lambda^{*}$ and a method of finding explicit solutions to (2.1). Each solution to (2.4) gives a distinct solution to (2.1), so we may convert the problem of counting solutions to the differential equation (2.1) to finding solutions of the equation (2.4). We postpone the proof of lemma 2.1 and theorem 2.2 in favor of giving two examples that illustrate how the theorem can be used. 


\subsection{An example computing $\lambda^{*}$}

Consider the case $g(s):=e^{s}$ and $\Omega:=(0,1)$. We claim that $\lambda^{*}=8 L^{2}$, where $L \approx 0.66274$ is the Laplace limit constant, the unique real solution to the equation

$$
\frac{L \exp \left(\sqrt{1+L^{2}}\right)}{1+\sqrt{1+L^{2}}}=1
$$

Numerical simulations show that $\lambda^{*} \approx 3.51383 \approx 8 L^{2}$, and if we plot against $2 H_{k}\left(G^{-1}(k)\right)^{2}$ we find a convincing confirmation.

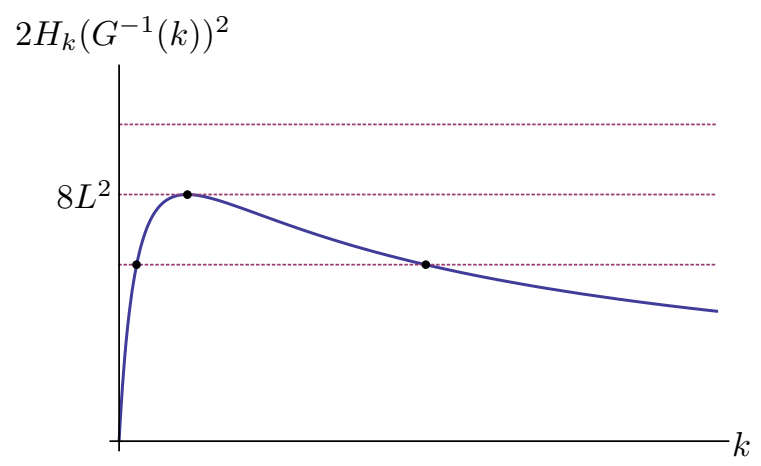

Figure 1: Counting solutions of $-\phi^{\prime \prime}=\lambda e^{\phi}$. Each intersection corresponds to a solution of (2.1), two for the lowest line, exactly one for $8 L^{2}$, and none above.

We proceed to prove what figure 1 suggests. We compute

$$
G(u)=\int_{0}^{u} e^{s} d s=e^{u}-1, \quad G^{-1}(k)=\log (k+1)
$$

and

$$
\begin{aligned}
H_{k}(y) & =\int_{0}^{y} \frac{d u}{\sqrt{k-\left(e^{u}-1\right)}} \\
& =\frac{2}{\sqrt{k+1}}\left(\operatorname{arctanh} \sqrt{\frac{k}{k+1}}-\operatorname{arctanh} \sqrt{\frac{k-\left(e^{y}-1\right)}{k+1}}\right)
\end{aligned}
$$

so

$$
H_{k}\left(G^{-1}(k)\right)=\frac{2}{\sqrt{k+1}} \operatorname{arctanh} \sqrt{\frac{k}{k+1}} .
$$

Differentiating in $k$ gives

$$
\frac{d}{d k}\left[H_{k}\left(G^{-1}(k)\right)\right]=\frac{1}{(1+k)^{3 / 2}}\left(\sqrt{\frac{k+1}{k}}-\operatorname{arctanh} \sqrt{\frac{k}{k+1}}\right)
$$

which is 0 when

$$
\sqrt{\frac{k+1}{k}}=\operatorname{arctanh} \sqrt{\frac{k}{k+1}}
$$


Setting $w:=\sqrt{\frac{k+1}{k}}$ we have $w=\operatorname{coth} w$ and $k=\frac{1}{w^{2}-1}$. We note that the equation $w=\operatorname{coth} w$ has a unique positive solution because $w \longmapsto w$ is increasing from 0 to $\infty$, while $w \longmapsto \operatorname{coth} w$ is decreasing. Thus we have uniquely determined a $w$ and corresponding $k$. Plugging this $k$ back in, we find

$$
H_{k}\left(G^{-1}(k)\right)=\frac{2}{w \sqrt{k}} \operatorname{arctanh} \frac{1}{w}=\frac{2}{\sqrt{k}}=2 \sqrt{w^{2}-1}
$$

so by theorem 2.2

$$
\lambda^{*}=\frac{2}{1-0}\left(2 \sqrt{w^{2}-1}\right)^{2}=8\left(w^{2}-1\right) .
$$

In order that $\lambda^{*}=8 L^{2}$ we need that $L=\sqrt{w^{2}-1}$. We check that $\sqrt{w^{2}-1}$ satisfies the defining property of $L$,

$$
\begin{aligned}
& 1=\frac{\sqrt{w^{2}-1} \exp \left(\sqrt{1+\left(\sqrt{w^{2}-1}\right)^{2}}\right)}{1+\sqrt{1+\left(\sqrt{w^{2}-1}\right)^{2}}} \\
& \Longleftrightarrow 1+w=\sqrt{w^{2}-1} \exp (w) \\
& \Longleftrightarrow(w+1)^{2}=\left(w^{2}-1\right) \exp (2 w) \\
& \Longleftrightarrow \exp (w)(w-1)=\exp (-w)(w+1) \\
& \Longleftrightarrow \operatorname{coth} w=w
\end{aligned}
$$

which we know is true, hence our claim follows.

\subsection{An example with no solution for $\lambda=\lambda^{*}$}

In this section we show that it is possible for no nonnegative solution to (2.1) with $\lambda=\lambda^{*}$ to exist. Take $g(s):=1+2 s$ and $\Omega:=(0,1)$. We start with intuition and then use the theorem 2.2 to prove our claim rigorously. The ODE in question is

$$
-\phi^{\prime \prime}=\lambda(1+2 \phi)
$$

and the "general solution" of $(2.5)$ is

$$
\phi(x)=c_{1} \cos (\sqrt{2 \lambda} x)+c_{2} \sin (\sqrt{2 \lambda} x)-\frac{1}{2} .
$$

Solving for the constants by applying boundary conditions gives

$$
\phi(x)=\frac{1}{2}(\cos (\sqrt{2 \lambda} x)+(\csc (\sqrt{2 \lambda})-\cot (\sqrt{2 \lambda})) \sin (\sqrt{2 \lambda} x)-1)
$$

from which it becomes apparent that problems begin occurring for the existence of nonnegative solutions whenever $\lambda \geq \frac{\pi^{2}}{2}$. Note that (2.6) is valid for $\lambda$ not of the form $k^{2} \frac{\pi^{2}}{2}$ for some $k \in \mathbb{N}$ and it does give solutions to (2.1) for arbitrarily large $\lambda$. However, those solutions with $\lambda>\frac{\pi^{2}}{2}$ admit negative values. We plot these solutions for different values of $\lambda$ in figure 2. As the figure shows, it appears that solutions to $-\phi^{\prime \prime}=\lambda(1+2 \phi)$ have fundamentally different behavior for $\lambda \in\left(k^{2} \frac{\pi^{2}}{2},(k+1)^{2} \frac{\pi^{2}}{2}\right)$ for different values of $k \in \mathbb{N}$. In particular, from the figure and from the 

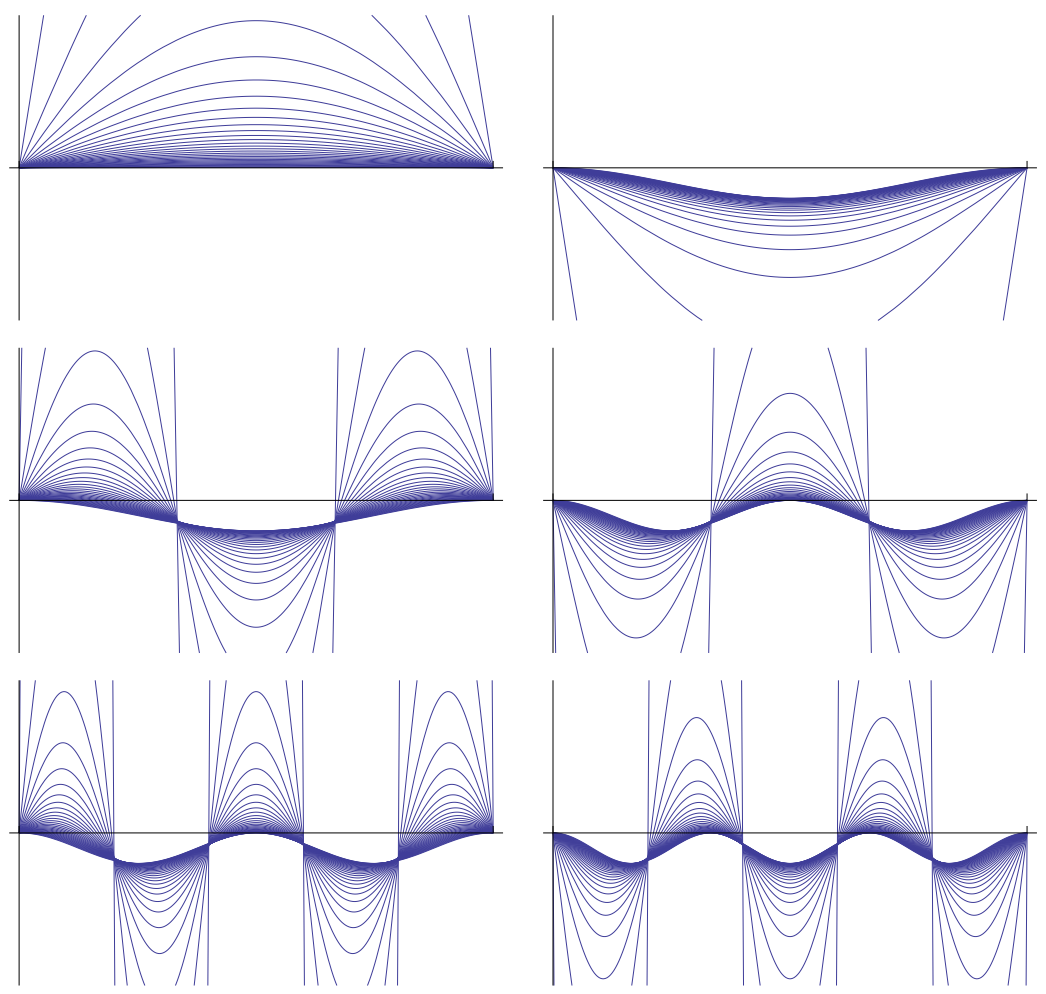

Figure 2: Sample solutions to $-\phi^{\prime \prime}=\lambda(1+2 \phi)$ plotted on $(0,1)$ with $\lambda \in\left(k^{2} \frac{\pi^{2}}{2},(k+1)^{2} \frac{\pi^{2}}{2}\right)$ for $k \in\{0,1,2,3,4,5\}$.

form of the solution (2.6) we see that nonnegative solutions blow up to $\infty$ as $\lambda \nearrow \frac{\pi^{2}}{2}$, so we expect that $\lambda^{*}=\frac{\pi^{2}}{2}$ and that there is no solution with $\lambda=\lambda^{*}$. This viewpoint is good for intuition, but not as strong as theorem 2.2. Since we do not necessarily have uniqueness of solutions to boundary value problems, even in the linear second order constant coefficients case, we cannot argue that (2.6) admitting negative values for some $\lambda$ implies there are no nonnegative solutions for that $\lambda$, nor can we use the fact that the formula (2.6) doesn't make sense for $\lambda=\frac{\pi^{2}}{2}$ to conclude that there is no nonnegative solution for $\lambda=\frac{\pi^{2}}{2}$. However, theorem 2.2 is strong enough to imply these claims. We proceed to confirm our intuition by applying theorem 2.2 to show $\lambda^{*}=\frac{\pi^{2}}{2}$ and that there is no nonnegative solution with $\lambda=\lambda^{*}$. As in the previous subsection, we find after some calculation that

$$
H_{k}\left(G^{-1}(k)\right)=\frac{\pi}{2}-\operatorname{arccot}(2 \sqrt{k})
$$

so that by theorem 2.2

$$
\lambda^{*}=\frac{2}{1-0}\left(\frac{\pi}{2}\right)^{2}=\frac{\pi^{2}}{2}
$$

However, $k \longmapsto H_{k}\left(G^{-1}(k)\right)$ is strictly increasing to $\frac{\pi}{2}$, but it never attains the value $\frac{\pi}{2}$. Thus by the second part of theorem 2.2 there are no nonnegative solutions to (2.1) with $\lambda=\lambda^{*}$. 


\subsection{Proof of theorem 2.2}

Proof of lemma 2.1. Suppose that $\phi$ solves (2.1). The setup of the problem implies that we have $\phi \in C([a, b]) \cap C^{2}((a, b))$. Then for $a<s<t<b$ we have

$$
\phi^{\prime}(t)=\phi^{\prime}(s)+\int_{s}^{t} \lambda g(\phi(r)) d r .
$$

Since $g \circ \phi$ is continuous on $[a, b]$ it follows that $\phi^{\prime}(t) \rightarrow \ell$ as $t \rightarrow b^{-}$for some $\ell \in \mathbb{R}$. Then L'Hôpital's rule implies $\phi$ is differentiable at $b$ with $\phi^{\prime}(b)=\ell$. In turn,

$$
\frac{\phi^{\prime}(b)-\phi^{\prime}(s)}{b-s}=\frac{1}{b-s} \int_{s}^{b} \lambda g(\phi(r)) d r \rightarrow \lambda g(\phi(b))
$$

as $s \rightarrow b^{-}$, so that $\phi^{\prime \prime}(b)=\lambda g(\phi(b))$. We apply a similar argument for $\phi^{\prime \prime}(a)$ to show $\phi \in C^{2}([a, b])$ and that $-\phi^{\prime \prime}=\lambda g(\phi)$ holds on all of $[a, b]$. If $g \in C^{k}([0, \infty))$ we may then bootstrap the fact that $\phi \in C^{k+2}([a, b])$ by observing that $\phi \in C^{n}([a, b])$ and $\phi^{\prime \prime}=-\lambda g(\phi)$ in $[a, b]$ implies $\phi \in C^{n+2}([a, b])$ for any $n \in \mathbb{N}$ with $n \leq k$.

Proof of theorem 2.2. Suppose that $\phi$ is a nonnegative solution of (2.1). By lemma 2.1 we know that $\phi \in C^{2}([a, b])$ and $-\phi^{\prime \prime}=\lambda g(\phi)$ on $[a, b]$. Multiplying (2.1) by $\phi^{\prime}$ we find

$$
\frac{d}{d s}\left[-\frac{1}{2} \phi^{\prime}(s)^{2}\right]=\frac{d}{d s}[\lambda G(\phi(s))]
$$

Integrating from $a$ to $x \in[a, b]$ then gives

$$
-\frac{1}{2}\left(\phi^{\prime}(x)^{2}-\phi^{\prime}(a)^{2}\right)=\lambda(G(\phi(x))-G(\phi(a)))=\lambda G(\phi(x))
$$

where here we imposed the boundary condition $\phi(a)=0$ along with $G(0)=0$. Rearranging and solving for $\phi^{\prime}(x)$ we find that for every $x \in[a, b]$

$$
\phi^{\prime}(x)=\sqrt{\phi^{\prime}(a)^{2}-2 \lambda G(\phi(x))} \text { or } \phi^{\prime}(x)=-\sqrt{\phi^{\prime}(a)^{2}-2 \lambda G(\phi(x))}
$$

where the choice potentially depends on $x$. We know that $\phi^{\prime \prime}=-\lambda g(\phi)<0$ so it follows that $\phi^{\prime}(a)>0$ since $\phi \geq 0$. Similarly $\phi^{\prime}(b)<0$ and so $\phi$ 's maximum occurs in $(a, b)$ and is unique. Call $x^{*} \in(a, b)$ the unique point such that $\max _{x \in[a, b]} \phi(x)=\phi\left(x^{*}\right)$. Thus the sign of $\phi^{\prime}$ does depend on $x$,

$$
\phi^{\prime}(x)=\left\{\begin{aligned}
\sqrt{\phi^{\prime}(a)^{2}-2 \lambda G(\phi(x))} & x \in\left[a, x^{*}\right], \\
-\sqrt{\phi^{\prime}(a)^{2}-2 \lambda G(\phi(x))} & x \in\left[x^{*}, b\right]
\end{aligned}\right.
$$

for all $x \in[a, b]$. Plugging in $x^{*}$ tells us that

$$
\max _{x \in[a, b]} \phi(x)=G^{-1}\left(\frac{\phi^{\prime}(a)^{2}}{2 \lambda}\right) .
$$

Continuing from (2.7), for $s \neq x^{*}$ we have $\sqrt{\phi^{\prime}(a)^{2}-2 \lambda G(\phi(s))} \neq 0$ and may divide to find

$$
\frac{\phi^{\prime}(s)}{\sqrt{\phi^{\prime}(a)^{2}-2 \lambda G(\phi(s))}}=1_{\left[a, x^{*}\right]}(s)-1_{\left[x^{*}, b\right]}(s) .
$$


Integrating over $[a, b]$ gives

$$
0=\left(x^{*}-a\right)-\left(b-x^{*}\right)
$$

so that $x^{*}=\frac{a+b}{2}$ as expected. Recall that $T(x)=\frac{b-a}{2}-\left|x-\frac{b+a}{2}\right|$ is the triangular bump with $T(a)=T(b)=0$ and $T\left(x^{*}\right)=T\left(\frac{b+a}{2}\right)=\frac{b-a}{2}$. Define the temporarily useful

$$
F(y):=\int_{0}^{y} \frac{d u}{\sqrt{\phi^{\prime}(a)^{2}-2 \lambda G(u)}} .
$$

We may integrate (2.9) from $a$ to $x \in[a, b]$ which gives

$$
F(\phi(x))=\int_{0}^{\phi(x)} \frac{d u}{\sqrt{\phi^{\prime}(a)^{2}-2 \lambda G(u)}}=T(x) .
$$

Since $g \in C([0, \infty))$ we know $G \in C^{1}([0, \infty))$, so that $F \in C^{2}\left(\left[0, G^{-1}\left(\frac{\phi^{\prime}(a)^{2}}{2 \lambda}\right)\right)\right)$ with $F^{\prime}>0$. Thus $F$ is invertible and so for $k:=\frac{\phi^{\prime}(a)^{2}}{2 \lambda}$

$$
\phi(x)=F^{-1}(T(x))=H_{k}^{-1}(\sqrt{2 \lambda} T(x))
$$

must be the form of the solution. Then by (2.8) and (2.10) we have that

$$
H_{k}\left(G^{-1}(k)\right)=H_{k}\left(\phi\left(x^{*}\right)\right)=\sqrt{2 \lambda} F\left(\phi\left(x^{*}\right)\right)=\sqrt{2 \lambda} \frac{b-a}{2}
$$

so that indeed (2.4) is satisfied.

Now that we know what the form of the solution must be, we attempt to verify when (2.11) actually solves (2.1). Suppose we use (2.11) to define $\phi$. We have implicitly fixed $\phi^{\prime}(a)$, which appears in the formula for $F$, so we cannot use this to define $\phi$. However, by replacing $\phi^{\prime}(a)$ with a constant $c>0$ in the formula, we may use (2.11) as a definition. With this definition of $\phi$, we indeed find by the inverse function theorem that $\phi^{\prime}(a)=\frac{1}{F^{\prime}\left(F^{-1}(0)\right)}=c$ so there is no ambiguity. That is, we have already shown that the form of $\phi$ must be (2.11), and we need only verify that if $k$ solves (2.4), then the choice $\phi^{\prime}(a):=\sqrt{2 \lambda}$ produces a solution of $(2.1)$.

Fix a $k$ solving (2.4). Since $F^{-1}$ is increasing, we know the maximum of $\phi$ occurs at the same maximum $x^{*}=\frac{b+a}{2}$ of $T$, i.e.

$$
\max _{x \in[a, b]} \phi(x)=H_{k}^{-1}\left(\sqrt{2 \lambda} \frac{b-a}{2}\right)=H_{k}^{-1}\left(H_{k}\left(G^{-1}(k)\right)\right)=G^{-1}(k)
$$

where in the second equality we used (2.4). We quickly note that

$$
\begin{aligned}
H_{k}\left(G^{-1}(k)\right) & =\int_{0}^{G^{-1}(k)} \frac{d u}{\sqrt{k-G(u)}} \\
& \leq \int_{0}^{G^{-1}(k)} \frac{d s}{\sqrt{k-\frac{k}{G^{-1}(k)}} s} \\
& =\frac{2 G^{-1}(k)}{\sqrt{k}}
\end{aligned}
$$


by convexity of $G$, which follows from the fact that $G^{\prime}=g$ is continuous and increasing. We note that the contents of the square root are indeed positive in the previous line for $s<G^{-1}(k)$. So in fact $F$ is bounded and therefore continuous at $G^{-1}(k)$ by the monotone convergence theorem. Hence $F^{-1}$ is continuous at $F\left(G^{-1}(k)\right)=\frac{b-a}{2}$. Moreover, the inverse function theorem gives us that $F^{-1} \in C^{2}\left(\left[0, \frac{b-a}{2}\right)\right)$ and so we immediately have that $\phi$ is $C^{2}$ except possibly at $x^{*}$. But

$$
F^{\prime}(u)=\frac{1}{\phi^{\prime}(a)^{2}-2 \lambda G(u)} \rightarrow \infty
$$

as $u \nearrow G^{-1}(k)$, so by the mean value theorem

$$
\begin{aligned}
\left(F^{-1}\right)^{\prime}\left(\frac{b-a}{2}\right) & =\lim _{y \rightarrow \frac{b-a}{2}} \frac{F^{-1}\left(\frac{b-a}{2}\right)-F^{-1}(y)}{\frac{b-a}{2}-y} \\
& =\lim _{x \rightarrow G^{-1}(k)} \frac{G^{-1}(k)-x}{F\left(G^{-1}(k)\right)-F(x)} \\
& =\lim _{x \rightarrow G^{-1}(k)} \frac{1}{F^{\prime}\left(\xi_{x}\right)} \\
& =0
\end{aligned}
$$

where $\xi_{x}$ is provided by the mean value theorem. This tells us that we have $F^{-1} \in C^{1}\left(\left[0, \frac{b-a}{2}\right]\right)$. We use a similar argument for $\left(F^{-1}\right)^{\prime \prime}$, by L'Hôpital's rule we have

$$
\begin{aligned}
\left(F^{-1}\right)^{\prime \prime}\left(\frac{b-a}{2}\right) & =\lim _{y \rightarrow \frac{b-a}{2}} \frac{\left(F^{-1}\right)^{\prime}\left(\frac{b-a}{2}\right)-\left(F^{-1}(y)\right)^{\prime}}{\frac{b-a}{2}-y} \\
& =\lim _{x \rightarrow G^{-1}(k)} \frac{0-\frac{1}{F^{\prime}(x)}}{F\left(G^{-1}(k)\right)-F(x)} \\
& \stackrel{L^{\prime} H}{=} \lim _{x \rightarrow G^{-1}(k)} \frac{-F^{\prime \prime}(x) / F^{\prime}(x)^{2}}{F^{\prime}(x)} \\
& =\lim _{x \rightarrow G^{-1}(k)} \frac{-F^{\prime \prime}(x)}{F^{\prime}(x)^{3}} \\
& =\lim _{x \rightarrow G^{-1}(k)} \frac{-\lambda g(x)\left(\phi^{\prime}(a)^{2}-2 \lambda G(x)\right)^{-3 / 2}}{\left(\phi^{\prime}(a)^{2}-2 \lambda G(x)\right)^{-3 / 2}} \\
& =-\lambda g\left(G^{-1}(k)\right) .
\end{aligned}
$$

A simple calculation from the inverse function theorem shows that $\left(F^{-1}\right)^{\prime \prime}(x) \rightarrow-\lambda g\left(G^{-1}(k)\right)$ as $x \nearrow G^{-1}(k)$, and therefore we also have $F^{-1} \in C^{2}\left(\left[0, \frac{b-a}{2}\right]\right)$. Since we know $F^{-1}$ is regular enough on its domain, we are now equipped to show $\phi=F^{-1} \circ T$ is $C^{2}$ at $x^{*}$. Restricting to $\left[a, x^{*}\right]$ or to $\left[x^{*}, b\right]$ gives us that $\phi \in C^{2}\left(\left[a, x^{*}\right]\right) \cap C^{2}\left(\left[x^{*}, b\right]\right)$, so we need only check that the left and right hand limits of derivatives match in order to show $\phi \in C^{2}([a, b])$. We have

$$
\phi^{\prime}(x)=\left(F^{-1}\right)^{\prime}(T(x)) T^{\prime}(x) \rightarrow 0
$$


as $x \rightarrow x^{*}$ since $T(x) \rightarrow T\left(x^{*}\right)=\frac{b-a}{2}$ as $x \rightarrow x^{*}$ and $\left|T^{\prime}(x)\right|=1$ for $x \neq x^{*}$. Furthermore,

$$
\begin{aligned}
\phi^{\prime \prime}(x) & =\left(F^{-1}\right)^{\prime \prime}(T(x))\left(T^{\prime}(x)\right)^{2}+\left(F^{-1}\right)^{\prime}(T(x)) T^{\prime \prime}(x) \\
& =\left(F^{-1}\right)^{\prime \prime}(T(x)) \\
& \rightarrow\left(F^{-1}\right)^{\prime \prime}\left(\frac{b-a}{2}\right)
\end{aligned}
$$

as $x \rightarrow x^{*}$ since $\left|T^{\prime}(x)\right|=1$ and $T^{\prime \prime}(x)=0$ for all $x \neq x^{*}$. Thus we have shown $\phi \in C^{2}([a, b])$. Easy calculations then verify that $-\phi^{\prime \prime}=\lambda g(\phi)$ on $[a, b]$.

At this point, we have completely classified solutions to (2.1) in terms of solutions to (2.4). From this correspondence, we claim that we can directly see the existence of and formula for the critical $\lambda^{*}$. Define $M(k):=H_{k}\left(G^{-1}(k)\right)$ for $k>0$ and $M(0):=0$. We will show that $M$ is continuous and bounded on $[0, \infty)$. This will imply $M([0, \infty))$ is a bounded interval containing 0 , which implies the existence of $\lambda^{*}$.

First we show $M$ is continuous on $[0, \infty)$. From above in $(2.12)$ we know that

$$
M(k) \leq \frac{2 G^{-1}(k)}{\sqrt{k}}=: B(k) .
$$

We consider what happens to $B(k)$ when $k \searrow 0$. Applying L'Hôpital's rule, we have

$$
\begin{aligned}
\lim _{k \rightarrow 0} \frac{2 G^{-1}(k)}{\sqrt{k}} & =\lim _{u \rightarrow 0} \frac{2 u}{\sqrt{G(u)}} \\
& =2 \sqrt{\lim _{u \rightarrow 0} \frac{u^{2}}{G(u)}} \\
& \stackrel{\mathrm{L}^{\prime} \mathrm{H}}{=} 2 \sqrt{\lim _{u \rightarrow 0} \frac{2 u}{g(u)}} \\
& =2 \sqrt{\frac{2 \cdot 0}{g(0)}} \\
& =0 .
\end{aligned}
$$

It follows that $M$ is continuous at 0 . Furthermore, for $k \in(0, \infty)$ the integrand in $M(k)$ is dominated,

$$
\frac{1_{s \leq G^{-1}(k)}}{\sqrt{k-G(s)}} \leq \frac{1_{s \leq G^{-1}(k)}}{\sqrt{k-\frac{k}{G^{-1}(k)}} s},
$$

where the integral of the right side, $B(k)$, satisfies dominated convergence as $k \rightarrow k_{0}$ for any $k_{0} \in(0, \infty)$. It follows by dominated convergence that $M$ is continuous on $(0, \infty)$. Hence $\{k: M(k)=\lambda\}$ is closed and bounded below by 0 , so it has a minimum if it is nonempty. In the case that the minimum exists for a given $\lambda$, it cannot be 0 since $0<\lambda$ so that $M(k)<\lambda / 2<\lambda$ in a neighborhood of 0 . For a fixed $\lambda$, sending $k \searrow 0$ amounts to sending $\phi^{\prime}(a) \searrow 0$. Since from (2.11) we see that $\phi(x)$ is strictly increasing in $\phi^{\prime}(a)$, the smallest valid $k$ will give 
a minimal solution. Thus, if there is a solution to (2.1), there is a minimal positive solution to (2.1).

Next we consider what happens to our bound $B(k)$ as $k \rightarrow \infty$. Since $g$ is increasing, convex, and not a constant, there exists an $s_{0} \geq 0$ such that $g_{+}^{\prime}\left(s_{0}\right)>0$, where $g_{+}^{\prime}$ denotes the right derivative of $g$. Then

$$
\begin{aligned}
\limsup _{k \rightarrow \infty} \frac{2 G^{-1}(k)}{\sqrt{k}} & =\limsup _{u \rightarrow \infty} \frac{2 u}{\sqrt{G(u)}} \\
& =\limsup _{u \rightarrow \infty} \frac{2 u}{\sqrt{\int_{0}^{u} g(s) d s}} \\
& \leq \limsup _{u \rightarrow \infty} \frac{2 u}{\sqrt{\int_{0}^{u}\left[g\left(s_{0}\right)+\left(s-s_{0}\right) g_{+}^{\prime}\left(s_{0}\right)\right] d s}} \\
& =\limsup _{u \rightarrow \infty} \frac{2 u}{\sqrt{u\left[g\left(s_{0}\right)-s_{0} g_{+}^{\prime}\left(s_{0}\right)\right]+\frac{u^{2}}{2} g^{\prime}\left(s_{0}\right)}} \\
& =\frac{2}{\sqrt{\frac{1}{2} g_{+}^{\prime}\left(s_{0}\right)}} \\
& <\infty
\end{aligned}
$$

which shows that $B(k)$ stays bounded as $k \rightarrow \infty$.

Thus the critical $\lambda^{*}$ exists, and moreover we have a formula for it:

$$
\lambda^{*}=\sup _{k>0}\left[\frac{2}{(b-a)^{2}} H_{k}\left(G^{-1}(k)\right)^{2}\right] .
$$

If we assume that $g_{+}^{\prime}(u) \rightarrow \infty$ as $u \rightarrow \infty$ we can conclude by $(2.13)$ that $M(k) \rightarrow 0$ as $k \rightarrow \infty$ as well. In this case, we know that the supremum for $\lambda^{*}$ is not attained by sending $k \rightarrow \infty$, and it follows that the supremum is actually a maximum and solution with $\lambda=\lambda^{*}$ exists. 


\section{References}

[1] S. Agmon, A. Douglis, and L. Nirenberg. Estimates near the boundary for solutions of elliptic partial differential equations satisfying general boundary conditions. i. Communications on Pure and Applied Mathematics, 12(4):623-727, 1959.

[2] H. Amann. On the existence of positive solutions of nonlinear elliptic boundary value problems. Indiana Univ. Math. J., 21:125-146, 1972.

[3] H. Berestycki, A. Kiselev, A. Novikov, and L. Ryzhik. The explosion problem in a flow. Journal d'Analyse Mathématique, 110(1):31-65, 2010.

[4] H. Berestycki, L. Nirenberg, and S. Varadhan. The principal eigenvalue and maximum principle for second-order elliptic operators in general domains. Communications on Pure and Applied Mathematics, 47(1):47-92, 1994.

[5] M. Crandall and P. Rabinowitz. Some continuation and variational methods for positive solutions of nonlinear elliptic eigenvalue problems. Archive for Rational Mechanics and Analysis, 58(3):207-218, 1975.

[6] L. Evans. Partial differential equations: Graduate studies in mathematics. American Mathematical Society, 2, 1998.

[7] M. Freidlin. Reaction-diffusion in incompressible fluid: asymptotic problems. Journal of differential equations, 179(1):44-96, 2002.

[8] M. Freidlin and A. Wentzell. Diffusion processes on graphs and the averaging principle. The Annals of probability, pages 2215-2245, 1993.

[9] A. Friedman. Partial differential equations of parabolic type, 1964. Holt, Reinhart, and Winston Inc., New York.

[10] M. Giaquinta and L. Martinazzi. An introduction to the regularity theory for elliptic systems, harmonic maps and minimal graphs. Edizioni della normale, 2005.

[11] D. Gilbarg and N. Trudinger. Elliptic partial differential equations of second order, volume 224. springer, 2001.

[12] G. Iyer, A. Novikov, L. Ryzhik, and A. Zlatoš. Exit times of diffusions with incompressible drift. SIAM Journal on Mathematical Analysis, 42(6):2484-2498, 2010.

[13] D. Joseph and T. Lundgren. Quasilinear dirichlet problems driven by positive sources. Archive for Rational Mechanics and Analysis, 49(4):241-269, 1973.

[14] L. Kagan, H. Berestycki, G. Joulin, and G. Sivashinsky. The effect of stirring on the limits of thermal explosion. 1997.

[15] T. Kato. Perturbation theory for linear operators, volume 132. springer, 1995.

[16] J. Keener and H. Keller. Positive solutions of convex nonlinear eigenvalue problems. Journal of Differential Equations, 16(1):103-125, 1974. 
[17] O. Ladyzenskaja. Linear and quasilinear equations of parabolic type. Transl. Math. Monographs, 23, 1968.

[18] R. Mennicken and M. Möller. Non-self-adjoint boundary eigenvalue problems, volume 192. Gulf Professional Publishing, 2003.

[19] A. Novikov. On the explosion problem in a ball.

[20] M. Reed and B. Simon. Methods of Modern Mathematical Physics: Vol.: 1.: Functional Analysis. Academic press, 1972.

[21] G. Rozenblum, M. Shubin, and M. Solomyak. Spectral theory of differential operators. In Partial differential equations VII, pages 1-235. Springer, 1994.

[22] D. Sattinger. Monotone methods in nonlinear elliptic and parabolic boundary value problems. Indiana University Mathematics Journal, 21(11):979-1000, 1972.

[23] J.-L. Thiffeault. Using multiscale norms to quantify mixing and transport. Nonlinearity, 25(2):R1, 2012.

[24] J. Vázquez and H. Brezis. Blow-up solutions of some nonlinear elliptic problems. Revista Matemática Complutense, 10(2):443, 1997. 